\title{
The Invisibility of Young Homosexual Women and Men with Intellectual Disabilities
}

\author{
Lotta Löfgren-Mårtenson
}

(C) Springer Science+Business Media, LLC 2008

\begin{abstract}
The aim of the article is to identify, describe and understand the opportunities and hindrances for young people with intellectual disabilities (ID) in expressing a variety of sexual expressions, such as homosexuality and bisexuality. The method is qualitative interviews with people with ID in the age of 16-27, staff members and parents. The study found young gay people with ID to be an invisible group, and that the possibilities to show a variety of sexual expressions are depending on the surroundings attitudes and behaviors toward them. Therefore it is important to teach about different sexualities in the sex education at special schools and also to do more research concerning homosexuality and bisexuality and intellectual disability, without having the heterosexual norm as a starting point.
\end{abstract}

Keywords Intellectual disability $\cdot$ Homosexuality $\cdot$ Bisexuality $\cdot$ Heterosexual norm $\cdot$ Sweden

\section{Background}

\section{Society's View of Different Sexualities}

Debates and discussions about sexuality today often use the term in the plural-sexualities - in order to stress that there is a wide spectrum of sexual preferences and orientations [1]. That such variety exists in society is seen by most as something unremarkable and expected. It is reasonable to expect that a range of different sexualities exist among people with disabilities. But a recent study about love and sexuality among young people with intellectual disabilities (ID) found that bisexuality or homosexuality was very unusual $[2,3]$. What can be the reason for this?

It is not possible to exactly quantify the number of people in any population who live in homosexual- or bisexual relationships or who identify as homosexual- or bisexual. A 